\title{
SECUENCIA ALUVIAL Y POST-ALUVIAL DE LA TERRAZA DE MUHARRA (CARMONA, SEVILLA): APORTACIÓN AL ACHELENSE EN EL VALLE DEL GUADALQUIVIR
}

\author{
ALLUVIAL AND POST-ALLUVIAL SEQUENCE AT THE MUHARRA \\ (CARMONA, SEVILLA) TERRACE. CONTRIBUTION TO THE RESEARCH \\ OF THE ACHEULIAN IN THE GUALDALQUIVIR BASIN
}

\author{
por \\ BAENA ESCUDERO, R. * \\ CARO GÓMEZ, J.A.**
}

RESUMEN Se presenta la secuencia estratigráfica aluvial y post-aluvial de la terraza de "Muharra" (T7) correspondiente al complejo de terrazas altas del Guadalquivir. En su formación se establecen dos episodios diferenciados, uno relacionado con la sedimentación del río y otro con formaciones hidromorfas y un paleosuelo fersialítico intercalado. El estudio de la abundante industria lítica recogida en la secuencia (más de 450 piezas), ha permitido definir dos horizontes paleolíticos: un yacimiento Achelense Final Transicional en un medio parcialmente confinado (nivel 4); y un horizonte con indicios de un posible Achelense sin determinar, en los niveles inferiores incluidos los propiamente aluviales.

ABSTRACT This paper shows the stratigrafhic alluvial and post-alluvial sequence of the "Muharra terrace" included into the High Terrace Complex of Guadalquivir river. Its origin is linked to two different episodies: one related to the sedimentation of the River; a second one, post-aluvial, with the development of hydromorphic processes and a insert paleo-soil. The research of the abundant lithic industry collected in that secuence (more than 450 pieces), allowed us to clasify it, as a deposit of Transitional Achalense, placed in a confined environment (level 4). The secuence shows signs that might correspond to "achalense" in general terms, in the lower levels (included the aluvial ones).

\footnotetext{
* Departamento de Geografía Física y Análisis Geográfico regional. Facutad de Geografía e Historia.

** Proyecto Formaciones Cuaternarias y Secuencia Paleolítica del Medio y Bajo Guadalquivir. Grupo de Investigación "Cuaternario y Geomorfología" (PAI, no 4079).
} 


\section{INTRODUCCIÓN}

En el último decenio las terrazas aluviales del Guadalquivir vienen siendo objeto de una investigación multidisciplinar a cargo de distintos Departamentos de la Universidad de Sevilla coordinada por los profesores F. Díaz del Olmo y E. Vallespí (1986, 89, y 92). Resultado de ello ha sido la elaboración de una cronosecuencia global para el Cuaternario de este río, donde se integran: tanto periodos estratigráficos convencionales, fechas absolutas, localizaciones y yacimientos paleolíticos; como los rasgos geomorfológicos más destacados a nivel de episodios aluviales, cambios de morfología, formaciones sedimentarias y suelos.

Plasmado todo ello en el marco de una Tesis Doctoral (Baena, 1993) y en la correspondiente Memoria del Proyecto "Formaciones Cuaternarias y Secuencia Paleolítica del Bajo Guadalquivir" que para la Consejería de Cultura (Junta de Andalucía) se encuentra en realización, su consideración permite la ubicación cartográfica, estratigráfica y cronológica, de cualquiera de las numerosas localizaciones y yacimientos arqueológicos que han sido y son, prospectados en la región.

La finalidad de este artículo radica en dar a conocer de manera sintética la aplicación de estos resultados globales a un yacimiento concreto como Muharra, recientemente descubierto por uno de nosotros (J.A. Caro) y el cual, de no ser por estos estudios previos, no supondría más que una nueva localización arqueológica puntual en un entorno aluvial como es el valle bajo del Guadalquivir.

Su elección como lugar de estudio radica en la abundante industria lítica en conexión estratigráfica aparecida (más de 450 piezas), todas ellas insertas en un contexto morfo-sedimentario que aporta nuevos datos para esclarecer las condiciones paleogeográficas acaecidas durante las distintas fases de ocupación paleolítica de la región del bajo Guadalquivir.

\section{EL SISTEMA DE TERRAZAS DEL GUADALQUIVIR Y SU IMPORTANCIA PARA EL ESTUDIO DEL PALEOLÍTICO Y LA GEOMORFOLOGÍA DEL CUATERNARIO}

El río Guadalquivir, con una cuenca hidrográfica de $57.377 \mathrm{Km} 2$ situada entre los $37^{\circ}$ y los $38^{\circ}$ de latitud Norte, constituye el principal colector del Sur de la Península Ibérica al tiempo que uno de los sistemas fluviales más importantes del dominio mediterráneo. En conjunto, la cuenca vertiente se articula sobre una depresión estructuralmente reciente y de nítido carácter postorogénico durante el Neógeno, orlada a Norte y Sur por sendos sistemas montañosos correspondientes a las estribaciones meridionales del zócalo hercínico (Sierra Morena) y los macizos alpinos de las Cordilleras Béticas (Fig.1).

Varios factores confluyen en este amplio espacio geográfico confiriéndole unas excepcionales condiciones para el estudio del Paleolítico y la geomorfología del Cuaternario. A saber:

- La gran extensión y continuidad de sus depósitos aluviales, particularmente en el valle medio y bajo, como resultado de una dilatada evolución geomorfológica que con posterioridad a los episodios de la regresión Neógena, abarcaría desde la implantación de los piedemontes de Sierra Morena (Baena et al., 1992; Recio et al., 1993) y los macizos subbéticos (Baena y Díaz del Olmo, 1994) hasta la configuración de la actual red hidrográfica. Esto es, todo el transcurso del Cuaternario en un sentido amplio (más de 2 M.a.). Se trata por tanto de un registro sedimentario de la suficiente continuidad temporal como para permitir valorar sobre él, tanto los distintos paleoambientes pleistocenos como el inicio y posterior evolución de los complejos tecnotipológicos del paleolítico de la región.

A ello se suma la enorme heterogeneidad de los aluvionamientos y las formaciones superficiales presentes en la zona, lo que necesariamente pasa por el establecimiento de interpretaciones dinámicas y valoraciones crono-estratigráficas diferenciadas en cada una de las terrazas. 
- En segundo lugar, la gran riqueza en restos paleolíticos relacionados con las terrazas. Estos comienzan a ser conocidos durante los años 50 a 70 mediante hallazgos puntuales (Saez, 1956 c.f. Martinez Santaolalla; Bordes y Viguier, 1969; Viguier y Thibault, 1973; Santonja y Querol, 1976 vid. 1988), para a partir de los años 80, con los trabajos preliminares de prospección de E. Vallespí y G. Alvarez, adquirir el grado de foco regional del paleolítico Inferior peninsular (Vallespí et al., 1982). Completa la sistematización de las investigaciones, la labor desempeñada en el marco del proyecto líneas arriba mencionado, al aportar numerosas localizaciones y yacimientos en conexión y ampliar considerablemente el número de piezas controladas. Se confirma así, el alcance de la región del Guadalquivir como modelo de secuencia cultural para el Paleolítico del Sur peninsular.

- Por último, el disponer en estos últimos años para el conjunto del valle, de un sistema de datación cronoestratigráfico con la suficiente resolución temporal como para abarcar la totalidad de sus episodios morfogenéticos. El método utilizado combina dataciones absolutas Uth con el análisis de las variaciones del campo magnético terrestre contenido en los sedimentos, permitiendo establecer la correspondiente correlación entre éste y la Escala temporal de magnetoporalidad de la Tierra (MPTS) (Baena, 1993; Baena y Díaz del Olmo, 1994).

\section{EL YACIMIENTO DE MUHARRA EN RELACIÓN CON LA SECUENCIA ALUVIAL DEL BAJO GUADALQUIVIR}

El Guadalquivir en su valle medio (Jaén y Córdoba) y bajo (Sevilla) cuenta con un sistema aluvial representado por 14 niveles de terrazas escalonadas y embutidas, agrupables en 4 conjuntos morfogenéticos denominados por nosotros (Díaz del Olmo et al., 1992) (Fig.2):

a) Altas Topografías aluviales y Complejo de Terrazas muy altas (T1 a T4): entre $+200-215 \mathrm{~m} \mathrm{y}+142-145 \mathrm{~m}$, únicamente se localizan en el valle medio a modo de retazos aislados, sin que por el momento se haya detectado industria lítica en conexión en ninguna de sus estratigrafías.

b) Complejo de Terrazas altas (T5 a T9): entre +130-139 m y +73-75 m, están presentes en todo el valle para su margen izquierda. En los sectores de Écija y La Campana sus aluvionamientos han arrojado varias localizaciones arqueológicas menores para las terrazas de $+110-115 \mathrm{~m}$ (T6) a +73-75 m (T9). Representan, hasta el momento, los testimonios iniciales de la secuencia paleolítica de Guadalquivir (Paleolítico Inferior indeterminado) con un escaso número de piezas integradas por núcleos irregulares y cantos tallados de filo unifacial.

c) Complejo de Terrazas Medias: representado por los niveles T10 (+45-55 m) a T12 (+35-40 m), se presentan oscilando entre ambas márgenes del valle. A nivel de aluvionamientos estos se muestran solapados, con gran potencia (entre 6 y más de $15 \mathrm{~m}$ ) y frecuentemente sobremontados por formaciones lacustres. Arqueológicamente los 2 niveles inferiores suponen el desarrollo del Achelense, de carácter pleno Ibérico en los yacimientos de la T11 con talla levallois, cantos tallados, bifaces, hendedores y triedros; y como Achelense final transicional asociado a restos paleontológicos de Elephas antiquus, Equus hydruntinus y Boss sp. en los aluvionamientos del siguiente nivel (T12).

d) Terrazas bajas y Complejo de terrazas muy bajas: por debajo de $+13-14 \mathrm{~m}$ (T14), corresponden a niveles embutidos de evolución Tardiglaciar-Holoceno. Entre sus características destacan el dominio de materiales finos a techo de barras de gravas y gravillas con abundante matriz arenosa, en ellas se han obtenido localizaciones menores correspondientes al Paleolítico Medio como final de la secuencia cultural.

A la vista de este contexto geomorfológico y cultural, el yacimiento de Muharra se inscribe en el bloque 
morfogenético de las terrazas altas del tramo bajo del Guadalquivir. Localizándose sobre la margen izquierda del río, en el sector comprendido entre Carmona y Sevilla, a la altura del Km 6,5 de la carretera Mairena del Alcor-Brenes (Fig.1).

Establecida la correspondiente cartografía geomorfológica a escala 1:25.000 de la zona (Baena, 1993), se observa que el sistema de terrazas en la transversal de Muharra se compone de 10 niveles entre $+110-115 \mathrm{~m}$, para el más alto (T6 de la secuencia general), y los 6-8 $\mathrm{m}$ de la actual llanura de inundación (Fig.3). En esta serie, el yacimiento estudiado se emplaza sobre la T7, caracterizada en el lugar por una disposición escalonada y fuertemente erosiva sobre el sustrato de margas azules.

En conjunto, los aluvionamientos de esta terraza no son muy potentes (1-2,5 m), salvo en aquellos sectores donde aparecen sobremontados por facies lacustres. Las series aluviales, no muy enérgicas, responden a secuencias sedimentarias positivas con barras de gravas basales, episodios cambiantes intermedios que denotan migraciones laterales de los cauces y niveles de finos en la parte superior.

\section{LA SECUENCIA SEDIMENTARIA DEL YACIMIENTO DE MUHARRA}

Entre la carretera de Mairena del Alcor y la hacienda Muharra, se ha podido reconocer una zanja de unos $100 \mathrm{~m}$ de longitud y $1,35 \mathrm{~m}$ de profundidad, orientada de Norte a Sur en disposición paralela al escarpe de la terraza. La secuencia estratigráfica observada se compone de muro a techo por (Fig.3):

1. Nivel de gravas basales de tamaño medio y carácter masivo, integradas por cuarcitas, grauwacas, areniscas de grano fino y algunos sílex. La parte inferior $(20 \mathrm{cms})$, subnivel $1 \mathbf{H}$, muestra rasgos hidromorfos representados por tonalidades verdes versicolores atribuibles a la proximidad del acuífero. Sobre él, se detectan los restos de un horizonte de suelo rojo (subnivel 1S) con pedolitos, en cuyos caracteres morfoedáficos, analizados sobre otro perfil próximo (San Pedro), resalta la abundancia de la caolinita y la esmectita; esta última en relación con los procesos de argiluviación (Baena, 1993). Hacia la parte superior, da paso a un depósito con abundante matriz limo-arcillosa y restos de carbonatos pulverulentos (subnivel 1L).

2. Con límite neto y erosivo sobre la unidad anterior y potencia variable $(40 \mathrm{cms}$ máximo), se desarrolla en disposición acuñada un depósito arcilloso de tonos verdes con presencia de niveles de limos finos, y gravas y gravillas que se organizan, bien dispersas en canales centimétricos, bien siguiendo el dispositivo de una red de grietas procedentes del techo de la formación.

3. Restos de un horizonte de suelo pardo-rojizo (5YR 6/4) de carácter planosólico $(15-20 \mathrm{~cm})$, presenta textura arenosa con marcada pérdida de elementos finos y gravillas $(\mathrm{L}=1,5)$ dispersas en la matriz hacia su parte superior. No obstante y en relación con las grietas existentes entre los prismas de la estructura, se detecta la presencia de gravillas algo mayores $(\mathrm{L}=3 \mathrm{cms})$.

4. Sobre la formación anterior, se desarrolla un depósito arcilloso de tonos pardo-negruzcos (10YR 4/3) con estructura prismática, grietas de retracción, y canales centimétricos dispersos. En las grietas es frecuente la aparición de gravillas y gravas verticalmente alineadas.

5. Finalmente cierra la unidad un depósito de entre 15 y $30 \mathrm{~cm}$ de color pardo, compacto, de textura areno-limosa con gravas y gravillas que representa el suelo de cultivo.

\section{ANÁLISIS Y VALORACIÓN DE LA INDUSTRIA LÍTICA}

En el estudio tipológico se ha seguido el plan de trabajo desarrollado en el Departamento de Prehistoria y Arqueología con el Prof. Vallespí, el método Bordes (Bordes, 1961) y la metodología propuesta por Santonja 
(Santonja, 1984 y 1984-85), con algunas apreciaciones propias concretadas en: la consideración de ciertas lascas como de "aspecto levallois" sin contabilizarse en los tipos correspondientes; y la clasificación dentro de los cantos tallados, de piezas que no desarrollan un filo.

La enumeración de las caracterísiticas de los distintos conjuntos líticos, se realizará siguiendo el orden establecido en la descripción de la secuencia estratigráfica.

\subsection{Gravas basales (Nivel 1, Fig.4, $\mathrm{n}^{0} 2-5$ )}

En él, la industria lítica se localiza en los subniveles inferiores (1H y 1S), mostrándose estéril hasta el momento el superior (1L). En el suelo rojo se han recogido 4 restos líticos compuestos por 2 lascas semicorticales y 2 núcleos, todos de cuarcita y sin rodamiento fluvial. Uno de los núcleos, con extracciones adyacentes, tiene una retalla que despeja un filo cortante a manera de canto tallado unifacial.

Respecto al subnivel inferior (1H), se han extraido 63 piezas líticas elaboradas en su totalidad sobre soportes de cuarcita a excepción de 2 que lo han sido en sílex. Según estén afectadas o no por rodamiento fluvial, hemos distinguido tres grupos:

a) Piezas con rodamiento fuerte (R3), constituidas por 3 lascas corticales y 1 núcleo, las longitudes de las primeras sobrepasan los $50 \mathrm{cms}$. El núcleo presenta una sola extracción.

b) Piezas que presentan huellas de rodamiento fluvial medio (R2); se trata de 1 lasca semi-cortical sin talón y 1 núcleo con dos extracciones opuestas más anchas que largas.

c) El último grupo diferenciado está compuesto por aquellas piezas que tienen un rodamiento fluvial "leve" o "dudoso".

Dado que este último grupo arroja una aceptable cantidad de industria (57 piezas), se ha podido realizar el correspondiente estudio tecno-tipológico que exponemos a continuación.

Hay un total de 23 lascas ( 2 en sílex y el resto en cuarcita) y 19 restos de talla ( 1 de sílex); de las lascas, 7 son corticales, 9 semicorticales y 7 internas. En cuanto a los talones, abundan los corticales $(56,2 \%)$, le siguen los lisos $(37,5 \%)$ y los diedros $(6,3 \%)$. Los bulbos reconocibles estan mayoritariamente muy marcados. Hay 10 lascas que han podido ser medidas ( $\mathrm{L}=46,4 ; \mathrm{A}=41,4 ; \mathrm{E}=16,8$ de media) resultando 4 más anchas que largas, manteniendo la misma tónica un tanto por ciento elevado de las fracturadas. Algunas de estas lascas presentan retoques marginales que no definen un tipo determinado. Hay 2 con aspecto levallois y una pequeña lasca de talla.

Se han recogido 2 núcleos, 1 con tres extracciones contiguas visibles $(62 \times 25 \times 36 \mathrm{~mm})$; el otro, es una lasca núcleo $(89 \times 63 \times 28 \mathrm{~mm})$ con una sola extracción.

El estudio tipológico de este mismo grupo comprende:

- En primer lugar hay que destacar la presencia de 1 punta levallois de cuarcita con la particularidad de terner el talón cortical.

- 2 cuchillos de dorso natural de cuarcita, filos recto y convexo con dimensiones de $58 \times 44 \times 17$ y $46 \times 26 \times 20$ $\mathrm{mm}$, respectivamente.

- 5 muescas, 4 simples y 1 retocada; ésta es inversa, amplia, profunda y lateral. Hay otra muesca inversa pero transversal. Las 3 restantes son directas, poco profundas y no muy amplias.

- Por último, hay 1 pieza que hemos calificado como diverso. Se trata de un útil elaborado sobre una gran lasca cortical de cuarcita que en el lado derecho posee dos levantamientos inversos y en el izquierdo otros que quedan cortados por una fractura longitudinal; entre estos y aquellos queda un filo cortante preexistente con aparentes huellas de uso. Es por ello, que lo hemos asociado a un posible útil afin a hendedor. 
En resumen, las características de la industria de este nivel se concretan en los siguientes aspectos generales: 1) Existencia de tres grupos diferenciados según su grado de rodamiento fluvial (R3, R2, y R1-R0), este último como más numeroso y representativo. 2) Estructura nada equilibrada desde un punto de vista tecnológico, ya que las piezas nodulares tienen una representatividad muy baja (9\%). 3) Elevada concentración de piezas $(50 \times \mathrm{m} 2)$. 4) La cuarcita es la materia prima predominante con tan solo un 3,2\% en sílex. 5) Presencia de técnica levallois. 6) En el utillaje predominan las muescas y los cuchillos de dorso natural. 7) Presencia de 1 útil afín a hendedor.

\subsection{Depósito arcilloso (Nivel 2. Fig. 5)}

Sobre la totalidad de la formación, se han extraido 59 restos líticos normalmente asociados a los canales y grietas existentes en ella.

\section{a) Estudio técnico.}

Comprenden un total de 21 lascas y 3 restos de talla, 2 de sílex y 22 de cuarcita; sólo uno de ellos presenta huellas de rodamiento fluvial. Se trata en la mayoría de los casos de lascas fracturadas afectando, en ocasiones, al bulbo. Las que se han podido medir arrojan los siguientes intervalos: $\mathrm{L}=60-28 \mathrm{~mm}, \mathrm{~A}=47-20 \mathrm{~mm}$ y $\mathrm{E}=35-10 \mathrm{~mm}$. Hay 6 lascas corticales, 11 semi-corticales y 4 internas. De los talones reconocibles, 5 son corticales, 2 lisos y 1 facetado. Varias tiene huellas aparentes de uso y en 6 existe retoque no definidor.

Los núcleos son 10 ( 1 en sílex, 1 en cuarzo y el resto en cuarcita) y sólo uno presenta rodamiento fluvial, siendo sus dimensiones medias de $\mathrm{L}=65, \mathrm{~A}=53 \mathrm{y} \mathrm{E}=40 \mathrm{~mm}$. De ellos, 8 presentan algunas extracciones contiguas, otro tiene extracciones desde múltiples planos y direcciones, y hay uno que resulta centrípeto sin preparación lateral y prácticamente agotado. A todo ello hay que añadir 5 restos nucleiformes de cuarcita.

b) Estudio tipológico.

Los utensilios registrados en este nivel han sido 20 , sin que ninguno esté elaborado sobre lasca levallois. Igualmente, no existe talón facetado ni diedro. Siempre se ha utilizado la cuarcita para su fabricación, excepto en un caso que lo ha sido el sílex; estando la mitad de los utiles, elaborados sobre lascas más anchas que largas.

El conjunto se desglosa en los siguentes tipos:

- 3 raederas, 1 en sílex transversal convexa y 2 sobre cara plana; el retoque en todas es simple.

- 1 cuchillo de dorso natural con filo convexo $(65 \times 52 \times 11 \mathrm{~mm})$.

- 8 muescas (6 simples y 2 retocadas) todas directas, las primeras no son muy amplias ni profundas. Las segundas son laterales y amplias con el retoque de una de ellas denticulado.

- 5 denticulados en su mayoría laterales, sólo uno transversal; el retoque es directo en 3 y alternante en 2 , estando sus dimensiones por encima de la media del nivel.

- 2 cantos tallados de cuarcita, 1 de ellos de filo unifacial, tiene una talla muy desarrollada en la linea de los bifaces parciales con aristas rectilíneas. El otro caso, se trata de un canto que denominamos truncado por tener un retoque que afecta desde una de sus caras a la otra con un ángulo de $90^{\circ}$. Es claro que en la talla no se ha buscado un filo, ni evidentemente, la extracción de lascas de esa medida, por no tener sentido. 
- Finalmente hay 1 pieza de cuarcita que posee una talla abrupta circular con un gran espesor, una retalla en sus lados y una base plana de deslizamiento que ha sido clasificada como cepillo; sus dimensiones son de $37 \times 35 \times 28 \mathrm{~mm}$.

En síntesis la serie del nivel 2, se caracteriza por: 1) Equilibrio tecnológico débil dado que los productos de talla, suponen uno o dos por cada núcleo, cuando éstos tienen una media de cuatro extracciones. 2) Predominio de las piezas sin rodamiento fluvial. 3) Empleo generalizado de la cuarcita como materia prima. 4) Indice levallois nulo. Predominio de los talones planos (lisos y corticales), con presencia de uno facetado. 5) Los nucleos poco elaborados son mayoritarios. 6) Dentro del utillaje dominan las muescas y el grupo de denticulados seguido por el musteriense. 7) Presencia aceptable de cantos tallados que suponen el $12 \%$ de los útiles. 8) Ausencia de utillaje bifacial, aunque un canto tallado está cerca de los bifaces parciales.

\subsection{Suelo pardo-rojizo (Nivel 3. Fig.4, nº1)}

En él, la industria encontrada, mayoritariamente de cuarcita (99\%) carece de rodamiento fluvial, siendo escasa y muy dispersa. Hay 8 lascas y 8 restos de talla; las lascas, normalmente de pequeño tamaño (L máxima $39 \mathrm{~mm}$ ), están en su mayoría fracturadas o les ha sido eliminado el bulbo, siendo 2 corticales, 1 semicortical y 5 internas con talones reconocibles planos.

Existen 3 núcleos y 2 restos nucleiformes; 2 de los núcleos tienen extracciones únicas apreciables y el otro corresponde a una lasca núcleo de donde se han extraído, al menos, tres pequeñas lascas y una más grande.

Perteneciente al grupo levallois hay una lasca atípica con talón diedro y algunos retoques.

Como útiles, se han identificado tres piezas: 1 bifaz sobre lasca de cuarcita con una talla no muy cuidada pero que despeja aristas bastante rectilíneas; su silueta no es regular, no obstante, por sus medidas y relaciones (80x63×30 mm.; m/e=2.1; $\mathrm{L} / \mathrm{m}=1,3 ; \mathrm{L} / \mathrm{a}=2,3 ; \mathrm{n} / \mathrm{m}=0,98)$, lo hemos clasificado como ovalado de talón . 1 canto tallado de filo unifacial que posee una acomodación lateral basal y un filo convexo bastante desarrollado con un claro retoque de regularización; sus medidas son $89 \times 80 \times 60 \mathrm{~mm}$. Finalmente 1 diverso, se trata de una pieza rota bien retocada con aristas algo sinuosas y lados simétricos, semejante a un fragmento de bifaz.

En resumen, este nivel se caracteriza por su escasez en restos líticos, la utilización de la cuarcita como materia prima dominante, el nulo rodamiento fluvial de la industria, la presencia de técnica levallois y la existencia de utillaje bifacial.

\subsection{Depósito arcilloso pardo-negruzco (Nivel 4. Figs. 6 y 7)}

Se trata del nivel con el mayor número de piezas (273) integradas por 110 lascas, 64 restos de talla, 50 núcleos y 49 utensilios; recogidas, en su mayor parte, en relación con grietas y pequeños canales.

a) Estudio Técnico.

Sobre un total de 110 lascas hemos comprobado que la mayoría de ellas, el 54,5\%, estan fracturadas y patinadas. Las fracturas pueden ser distales o laterales-oblícuas aunque son más numerosas las segundas afectando en muchas ocasiones a los bulbos. Casi la mitad de ellas resultan más anchas que largas siendo sus medidas en mm de: 


\begin{tabular}{cccccc}
\hline № & INTERVALO & MEDIA & MEDIANA & MODA & DESVIACION \\
\hline L 35 & $21-77$ & 39,9 & 38 & 30 & 13,55 \\
A 35 & $20-57$ & 36,7 & 38 & 40 & 9,16 \\
E 35 & $9-34$ & 15,9 & 15 & 15 & 5,88
\end{tabular}

El $45,4 \%$ son lascas internas, el $35,5 \%$ semicorticales y el $19,1 \%$ corticales con rodamientos nulo y excepcionalmete leve. Sólo hemos podido reconocer sesenta y nueve talones de los cuales cuarenta y seis son corticales y veintitres lisos; en los bulbos abundan los poco marcados, aunque no faltan los prominentes y alguno reflejado. La materia prima empleada en la elaboración de las lascas ha sido mayoritariamente la cuarcita con el $92,7 \%$ (7,3\% en sílex). Hay un buen número de lascas que presentan huellas aparentes de uso y otras retoque intencionado que no define ningún tipo concreto. Debris y restos de talla hemos identificado 64 (56 de cuarcita y 8 de sílex).

Los núcleos contabilizados en el nivel son 50 (19 restos indefinidos), elaborados en cuarcita (99\%) con las siguientes dimensiones:

\begin{tabular}{cccccc}
\hline № & INTERVALO & MEDIA & MEDIANA & MODA & DESVIACION \\
\hline L 31 & $39-110$ & 68,5 & 65 & - & 17,5 \\
A 31 & $31-102$ & 55,4 & 52 & 50 & 16,5 \\
E 31 & $19-70$ & 41,5 & 37 & 35 & 13,6
\end{tabular}

El desglose tecnológico arroja las siguientes categorias: 11 sólo tienen alguna extracción aislada; 12 presentan extracciones contiguas paralelas entre sí, estando 7 de ellos casi agotados; hay 1 con extracciones subparalelas que ocupan casi todo el contorno; también hay un sólo ejemplar que presenta extracciones desde mútiples planos; 2 núcleos muestran extracciones bifaciales a partir de una arista central (uno de ellos se asemeja a un canto tallado); hay 3 núcleos con extracciones centrípetas en una cara, 2 sin preparación periférica y el otro con preparación periférica parcial, son pequeños y están casi agotados; existe 1 pequeña lasca-núcleo en la cual se ha efectuado una sóla extracción sin preparación de plano de percusión.

b) Estudio Tipológico.

Los 49 utensilios identificados en este nivel se han englobado en los siguientes grupos característicos:

- 2 lascas levallois, una típica de cuarcita y otra atípica de sílex con algunos retoques; sus medidas son $42 \times 38 \mathrm{~mm}$ y $42 \times 31 \mathrm{~mm}$.

- 12 raederas, todas en cuarcita, las simples (7) corresponden a 2 rectilíneas, una de ellas con retoque simple no muy profundo y una pequeña muesca de usos, la otra presenta un retoque semiabrupto y profundo; las convexas (4) presentan retoque simple con una de ellas como la de mayor tamaño en la serie (105x80 mm.); la cóncava tiene retoque simple y el talón suprimido. La única raedera transversal aparecida es convexa con retoque simple. En lo que respecta a las raederas sobre cara plana (3), todas poseen retoque simple -aunque en una de ellas resulta bastante plano- con formato superior a la media. Y por último, hay 1 raedera de retoque alterno ladeada, ambos retoques son simples aunque uno tiende a marginal.

- 4 cuchillos de dorso atípico y 3 perforadores. Los primeros están fabricados sobre lascas de cuarcita de gajo de naranja con retoque simple parcial en el dorso. Los perforadores son atípicos ( 2 de cuarcita y 1 de sílex), con muesca en un lado y un retoque burdo en el otro. 
- 3 denticulados laterales, 2 convexos y 1 rectilíneo, elaborados sobre lascas internas que no conservan el bulbo.

- Existen además otros utensilios sobre lasca tales como: 3 cuchillos de dorso natural, uno de ellos con una de las mayores dimensiones de la serie $(93 \times 55 \mathrm{~mm})$. 14 muescas (13 en cuarcita y 1 en sílex) la mayoría simples (12), producidas seguramente por el uso, 8 son directas y 4 inversas; hay 2 retocadas, una elaborada sobre lasca de aspecto levallois, resulta directa y lateral; la otra es inversa y amplia.

- 1 diverso correspondiente a una lasca que posee un retoque simple distal.

- Dentro del macroutillaje hemos distinguido 7 piezas todas en cuarcita e integradas por:

5 cantos tallados, 4 unifaciales y 1 bifacial; todos tienen el filo simple aunque uno posee un diente en el centro; de los unifaciales 3 son convexos y 1 rectilíneo, extendiéndose la talla hasta la mitad del canto; el perfil de todos resulta rectilíneo. En el bifacial, el filo es simple, convexo y muy desarrollado. Sus dimensiones medias presentan los siguientes valores característicos en mm. y g.: $\mathrm{L}=73 ; \mathrm{A}=85 ; \mathrm{E}=48$ y $\mathrm{P}=398$.

1 bifaz espeso; al estar tallado completamente por una de sus caras y parte de la otra, lo consideramos parcial. Al orientarlo dejando la parte más estrecha en el extremo distal, resulta la base la zona más tallada; hecho muy anormal en las series estudiadas por nosotros en el Guadalquivir. De acuerdo con su morfología y valores característicos lo hemos clasificado como ovalado de talón $(L=129 ; m=89 ; e=52 ; L / a=2,15 ; n / m=1$; $\mathrm{m} / \mathrm{e}=1,2 ; \mathrm{L} / \mathrm{m}=1,5)$.

1 triedro elaborado sobre canto rodado aplanado de cuarcita, con una punta corta pero bien despejada y sus aristas cortantes y rectilíneas. Sus dimensiones son $\mathrm{L}=104 ; \mathrm{m}=72$ y e $=47 \mathrm{~mm}$ ).

En suma, la serie se caracteriza por los siguientes aspectos generales: 1) Estructura equilibrada en cuanto a los productos de talla sin retocar y los utensilios, así como en lo referente a la suma de ambos respecto a los núcleos y piezas nodulares (por cada pieza nodular se han recuperado 4 lascas, esta misma es la media de extracciones que se dan en los núcleos); por término medio, fueron retocadas 1 de cada 4 lascas. 2) Empleo generalizado de la cuarcita como materia prima, con presencia mínima del sílex. 3) Indices levallois y facetado amplio, muy bajos. 4) Reducido número de núcleos centrípetos y de preparación. 5) En los utensilios, el grupo característico más numeroso es el Musteriense seguido del grupo Paleolítico Superior integrado por útiles poco típicos y el grupo de denticulados; las muescas son el útil más numeroso, aunque las verdaderas muescas retocadas son escasas. 6) La presencia de cantos tallados no es muy amplia pero representativa, el índice sobrepasa 10.7) Indice bifacial muy bajo, algo más de 4.

\subsection{Valoración global del conjunto (Fig.8)}

El estudio de la industria aparecida en los diferentes niveles del yacimiento Muharra, debe hacerse de un modo conjunto dada la continuidad del contexto estratigráfico y el hecho de que sólo en uno de ellos (nivel 4), la serie recogida tiene el suficiente tamaño como para ser considerada representativa.

En función de los caracteres de la industria lítica analizada, deben distinguirse dos bloques diferenciados. Por un lado estaría la industria de los niveles 1,2 y 3 (147 piezas), y por otro la del nivel pardo-negruzco (273 piezas). En el primero apreciamos como no existe equilibrio tecnológico, puesto que los productos de talla representan un porcentaje muy elevado en comparación con los núcleos y útiles nodulares. Sin embargo, el segundo conjunto (nivel 4) alcanza una estructura bien equilibrada, con una cadena operativa en la que todos sus niveles estan significados, lo que sugiere junto con otros detalles (rodamiento, estructura sedimentaria del nivel, etc.), un posible emplazamiento original de esta industria. 
Para el conjunto de ambas series, nos encontramos ante industrias no levallois, con un índice aceptable de cantos tallados como característica común del yacimiento y del Paleolítico de las depresiones fluviales peninsulares. No obstante, a nivel intrínseco sí es posible marcar ciertas diferencias evolutivas entre la serie inferior, con núcleos poco elaborados, y la superior con un porcentaje importante de los centrípetos. En el mismo sentido apunta el estudio del utillaje, con un nivel 4 donde se manifiesta: un significativo aumento del grupo Musteriense, la aparición del grupo Paleolítico Superior, y nuevos tipos de retoque más amplios y delineación más cuidada.

Atendiendo a estas características tecno-tipológicas, incluido su bajo índice bifacial, parece adecuado considerar el conjunto superior (nivel 4) como Achelense; adecuándose perfectamente con los momentos finales de la secuencia regional atribuida al Achelense final transicional por el Prof. Vallespí (1994).

Respecto a la serie del grupo inferior (niveles 1,2 y 3), su interpretación resulta más complicada. En efecto, aunque la industria del suelo pardo-rojizo (nivel 3) puede adscribirse al Achelense por la presencia de un bifaz y de un fragmento de bifaz, no ocurre igual con los niveles inferiores ( 1 y 2), donde tan sólo, la existencia de un canto tallado unifacial muy desarrollado en el nivel 2 y un útil afín a hendedor en el nivel de gravas (1), constituyen, a nuestro entender, las únicas evidencias probables en las que argumentar una continuidad cultural hasta el início de la secuencia estratigráfica.

\section{INTERPRETACIÓN DE LA SECUENCIA DE LA TERRAZA Y DISCUSIÓN}

La comprensión del significado dinámico y paleogeográfico de la estratigrafía presente en la terraza de Muharra, se establece a partir de la diferenciación entre formaciones propias de la secuencia aluvial (nivel 1), y aquellas otras que integran la cobertera discordante como exponentes de una prolongada evolución postaluvial de la terraza (niveles 2,3 y 4 ).

La presencia de barras de gravas con tamaño medio y abundante matriz arcillosa (subnivel $1 \mathrm{H}$ ), selladas por los restos de un paleosuelo fersialítico parcialmente desmantelado (1S), constituyen el final del aluvionamiento que compone la terraza de Muharra. Su vinculación a un medio deposicional de carácter lateral respecto a la circulación paleohidrológica de la corriente del río en el momento de su formación, explica que la industria aparecida en ambos subniveles manifieste rodamientos diversos aunque predominando los leves. Los restos del suelo rojo (subnivel 1s) con los que culmina la serie anterior, garantizan el escaso desplazamiento de la industria en él inserta, por cuanto para su formación es indispensable la existencia de condiciones con la suficiente estabilidad morfogenétiça. Termina el proceso de sedimentación aluvial, con la incorporación de un depósito de baja energía (subnivel 1L) sobre el que se genera una nueva formación edáfica con aporte de carbonatos como muestra de la emersión del medio. Actualmente el techo de la terraza se muestra en su parte superior decapitado.

La disposición del nivel 2 en modo erosivo y acuñado sobre la unidad anterior a la que desmantela hasta alcanzar el nivel de gravas, supone una importante discordancia estratigráfica al tiempo que un hiatus temporal de la suficiente magnitud como para permitir la formación del anterior y su posterior erosión. A partir de aquí, tiene lugar una nueva dinámica paleogeomorfológica vinculada a la acción de drenajes superficiales que discurren sobre el techo de la terraza procedentes del nivel superior (T6) y Los Alcores.

Se genera así, un medio deposicional parcialmente confinado donde son habituales los encharcamientos permanentes con tendencia a su colmatación (arcillas verdes) con posterior drenaje mediante arroyadas superficiales de pequeña dimensión (canales de gravillas).

Son estos tipos de situaciones con las que se relacionan la aparición de los restos líticos, bien en relleno de grietas de retracción desarrolladas sobre el final de las colmataciones arcillosas, bien como arratres dentro de 
los canales superficiales. Termina este episodio con la formación de un suelo pardo-rojizo (nivel 3) vinculados al cual, se han extraido algunas piezas dispersas y carentes de rodamiento.

Sobre él, vuelve a repetirse el ciclo inicial con nuevo desarrollo de ambientes hidromorfos (depósito arcilloso pardo-negruzco) relacionados con zonas deprimidas y posteriormente drenadas.

A tenor de la cronosecuencia global del Guadalquivir (Fig.2), las formaciones sedimentarias que integran la terraza analizada se encuadran dentro de una doble horquilla cronológica. La primera, afecta a los depósitos aluviales (Nivel 1) petenecientes al complejo de terrazas altas ( $\mathrm{T} 7 \mathrm{a}+95-100 \mathrm{~m}$ ) y situados dentro de la magnetozona de polaridad normal Brunhes con cronología posterior a 600.000 B.P. y anterior a 300.000 B.P., tomando como referencia la proyección del evento Biwa, negativo, en facies lacustres del primer nivel de las terrazas medias ( $\mathrm{T} 10 \mathrm{a}+45-55 \mathrm{~m}$ ). Respecto a la segunda, la presencia de un suelo pardo-rojizo intercalado entre los niveles hidromorfos 2 y 4 , indica que, arrancando desde la referencia cronológica anterior, se prolonga hasta el Pleistoceno Superior (más de 80.000 B.P.).

En el conjunto estudiado, tan sólo el nivel 4 constituye, por su número de piezas y equilibrio de la cadena operativa, un verdadero yacimiento arqueológico del Paleolítico. La comparación de las características tecnotipológicas de su industria, en relación con la ya estudiada en los yacimientos de Antiguos Viveros (Vallespí, 1994) y Pionner J.A. (Caro, 1994) ambos en la terraza Jarillas (T12, +26-30 m), nos induce a considerarlas del mismo momento cultural (Achelense Final Transicional) con cronología entre 170.000 y 80.000 B.P (Baena, 1993).

El resto de la secuencia post-aluvial (niveles 3 y 2), representa sendas localizaciones con industria carentes de rodamiento y de adscripción achelense sin determinar. La primera de ellas, es la única aparecida en este tipo de formación (suelos fersialíticos) que se encuentra fosilizada (más de 170.000 B.P.). La segunda, dado su particular contexto sedimentario y el alto porcentaje de útiles y núcleos aparecidos, constituye un nivel potencial para la busqueda de un yacimiento "in situ".

Respecto a la localización del nivel aluvial, resaltar su importancia en el contexto de las terrazas altas, por cuanto hasta el momento, no se había encontrado un número tan elevado de piezas en la franja cultural del Paleolítico Inferior Indeterminado. Este horizonte quedaba explicitado a partir de las localizaciones menores de Motilla, Monclova, Cañada del Rosal y Calonge Alto (Fig.2), con un escaso número de restos líticos (lascas y núcleos) culturalmente no definidores. No obstante, en la T8 (La Viñuela) un bifaz rodado procedente del desmantelado de la terraza, hizo pensar en la posible adscripción Achelense de este nivel (Vallespí, 1994).

La aparición ahora de un útil afín a hendedor en el aluvionamiento, permitiría confirmar esta primera hipótesis, ampliando el horizonte cultural Achelense hasta la T7.

\section{CONCLUSIONES}

La génesis de esta terraza muestra dos episodios sedimentarios diferenciados separados por una discordancia y un hiatus temporal.

El inferior propiamente de carácter aluvial responde, en el contexto de la crono-secuencia del Guadalquivir, a un Pleistoceno Medio (600.000-300.000 B.P.). El superior, relacionado con escorrentías superficiales y medios confinados, abarcaría el tránsito Pleistoceno Medio-Superior y el Pleistoceno Superior.

El yacimiento de Muharra corresponde al nivel 4, como final de la secuencia post-aluvial de la T7. Define un momento cultural correspondiente al Achelense Final Transicional en medios con encharcamientos temporales y drenajes de pequeña entidad.

Las localizaciones situadas en los niveles 3 y 2 , presentan condiciones similares a otras ya conocidas en el Bajo Guadalquivir, con la particularidad de que, en este caso, se encuentran fosilizadas. Para el nivel aluvial, 
resaltar la importancia de la industria aparecida, al posibilitar la amplición del horizonte cultural achelense hasta el conjunto de terrazas altas.

Finalmente, el trabajo reafirma la importancia de las secuencias crono-estratigráficas globales en el estudio del Pleistoceno continental, permitiendo la ubicación de cualquier nuevo yacimiento, al tiempo que éstos aportan nuevos datos que depuraran y completan el marco geomorfológico y cultural del Paleolítico de la región.

\section{Agradecimientos}

Contribución al Proyecto DGICYT, PB93-0105.

A los Profesores E. Vallespí y F. Díaz del Olmo, por las sugerencias aportadas y la revisión de este trabajo.

Al propietario de la Estación de Servicios Santa Teresa (Carmona) D. Alfredo J. Rosa Fernández, por su amable colaboración al facilitarnos el acceso al yacimiento.

\section{BIBLIOGRAFÍA}

BAENA ESCUDERO, R.; DÍAZ DEL OLMO, F. y RECIO ESPEJO, J.M. (1992): "Valle medio del Guadalquivir: terrazas fluviales en la transversal Villa del Río (Córdoba)", VIII Reunión Nacional sobre Cuaternario, Valencia, AEQUA.

BAENA ESCUDERO, R. (1993): Evolución cuaternaria (3 M.a.) de la depresión del medio-bajo Guadalquivir y sus márgenes (Córdoba y Sevilla). Tesis Doctoral, 589 págs + cart. Univ. de Sevilla.

BAENA ESCUDERO, R. y DÍAZ DEL OLMO, F. (1994): "Cuaternario aluvial de la depresión del Guadalquivir: episodios geomorfológicos y cronología paleomagnética", Geogaceta, 15: 102-104.

BAENA ESCUDERO, R. y DÍAZ DEL OLMO, F. (1994): "Heterogeneidad de los piedemontes alpinos de los macizos subbéticos y depósitos asociados (Sevilla, Córdoba, Jaén)", Geomorfología en España, J.Arnáez, J.M.García Ruíz y A.Gómez Villar (Edrs.), Logroño. T.I:291-304.

BORDES, F. y VIGUIER, C. (1969): "Présence de galets taillés de type ancien dans la région de Carmona (province de Seville, Espagne)", C.R. Acad. Sc. Paris, 289, ser. D.:146-147.

BORDES, F. (1961): Typologie du Paleolithique Ancien et Moyen. Impr. Delmas. Bourdeaux.

CARO GÓMEZ, J.A. (1994): "Análisis de un perfil nuevo en terraza las jarillas (Pleistoceno Medio-Superior), Sevilla. Yacimiento Pionner J.A.”, Spal (Universidad de Sevilla), 2:303-309 (e.p.).

DÍAZ DEL OLMO, F.; VALLESPI, E. y ÁLVAREZ, G. (1986): "Formations superficielles détritiques et séquence paleolithique du Bas Guadalquivir (SW de l'Espagne)", Mediterranée 3:61-65.

DÍAZ DEL OLMO, F.; VALLESPÍ, E.; BAENA, R. Y RECIO, J.M. (1989): “Terrazas pleistocenas del Guadalqivir occidental: geomorfología, suelos, paleosuelos y secuencia cultural”, en El Cuaternario en Andalucía Occ., AEQUA Monografías 1:33-42.

DÍAZ DEL OLMO, F; VALLESPI, E. y BAENA, R. (1992): "Cuaternario y secuencia Paleolítica en las terrazas del bajo y medio Guadalquivir: aluvionamientos, coluviones, suelos y paleosuelos", en Investigaciones Arqueológicas en Andalucía (1985-1992), Huelva, 193-210.

RECIO ESPEJO, J.M.; CANO HENARES, M‥D.; BAENA ESCUDERO, R.; RODRÍGUEZ VIDAL, J. y DIAZ DEL OLMO, F. (1993): "Rañas en el borde meridional del Macizo Hespérico (Sierra Morena)", La Raña en España y Portugal, Monografías del Centro de Ciencias Medioambientales 2:3-13.

SÁEZ MARTíN, B. 1956: Noticiario Arqueológico Hispánico III y IV. Cuadernos 1-3 (1954-55):219-223 y 232. 
SANTONJA, M. y QUEROL, M.A. (1988): "Paleolítico Inferior arcaico de la Depresión del Guadalquivir". Trabajos de Paleolítico y Cuaternario. F. Díaz del Olmo y E. Vallespí (ed.), Universidad de Sevilla, 39-52. SANTONJA, M. y PÉREZ-GONZÁLEZ, A. (1984): "Las industrias Paleolíticas de la Maya I y su ámbito regional", E.A.E. 135, Ministerio del Cultura. Madrid.

SANTONJA, M. (1984-85): "Los núcleos de lascas en las industrias paleolíticas de la Meseta Española". Zephyrus, XXXVII-XXXVIII.

VALLESPÍ, E.; ÁLVAREZ, G.; AMORES, F.; y ESCACENA, J.L. (1982): “Complejo de cantos tallados y bifaces en el Bajo Guadalquivir: perspectivas de su estudio". Grupo Español de Trabajo del Cuaternario, 5 reunión. Universidad de Sevilla, 79-94.

VALLESPÍ, E. (1994): "El Bajo Guadalquivir en el Paleolítico Inferior y Medio peninsular”, Homenaje al Dr. Joaquín González Echegaray, Museo y Centro de Investigación de Altamira, Monografías, 17:13-16.

VIGUIER, C. y THIBAULT, C. (1973): "Nouveaux éléments de datation des formations de Piédmont de la Sierra Morena à l'Ouest de Sevilla". Est. Geológicos, 29:351-356. 


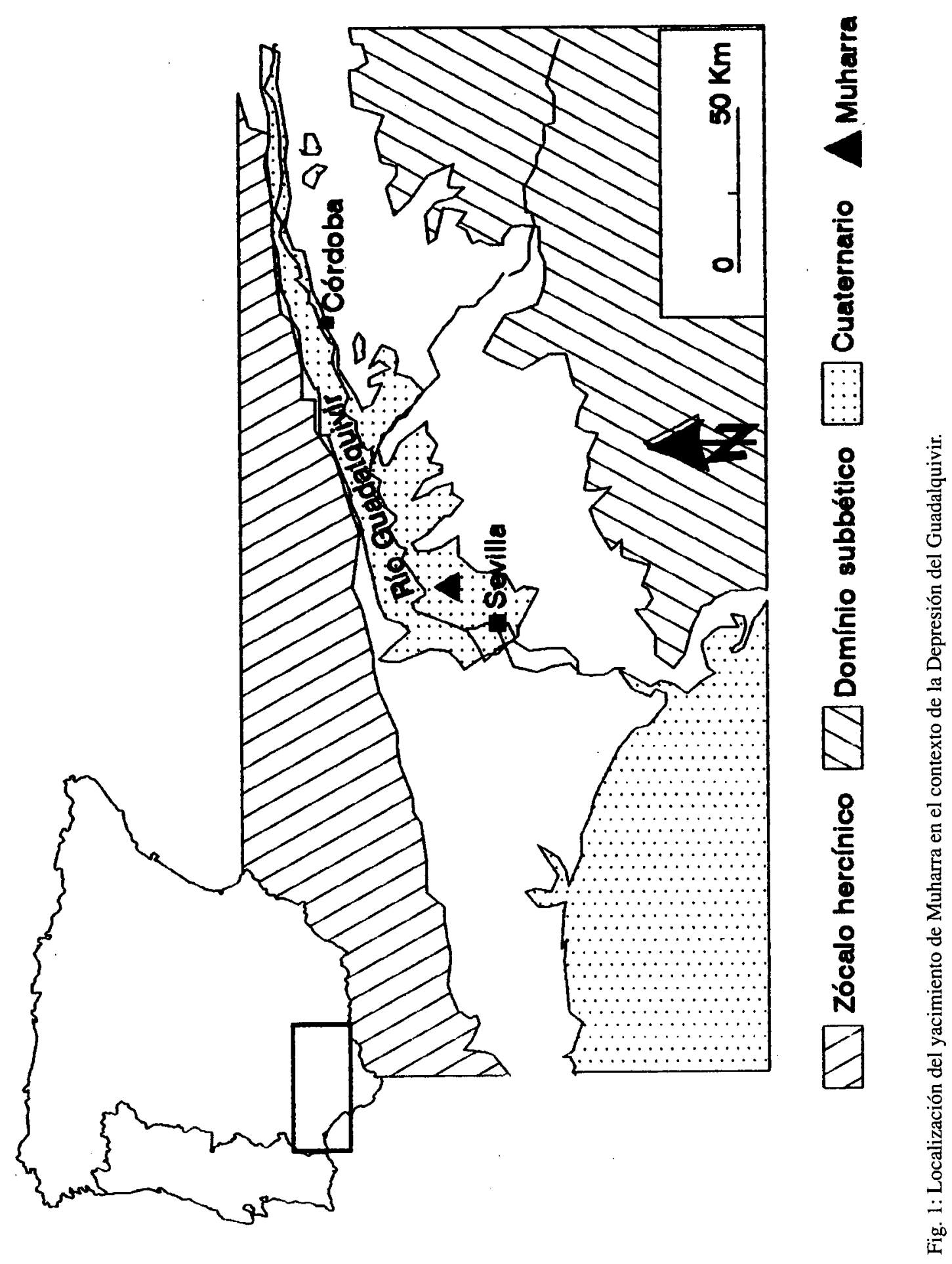




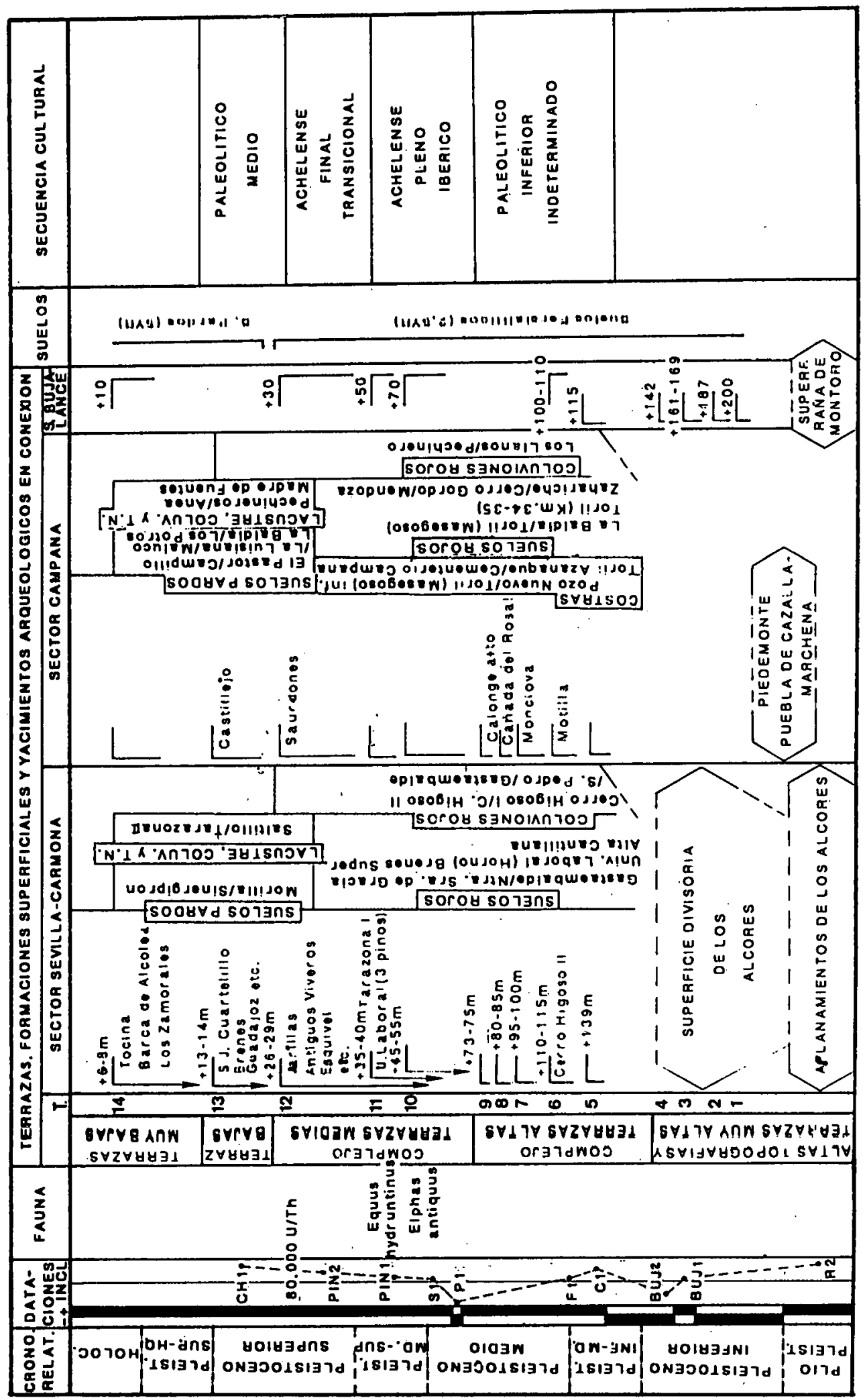

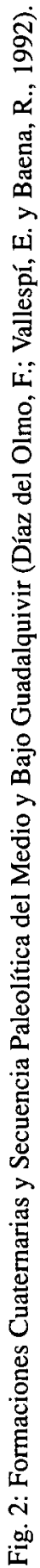




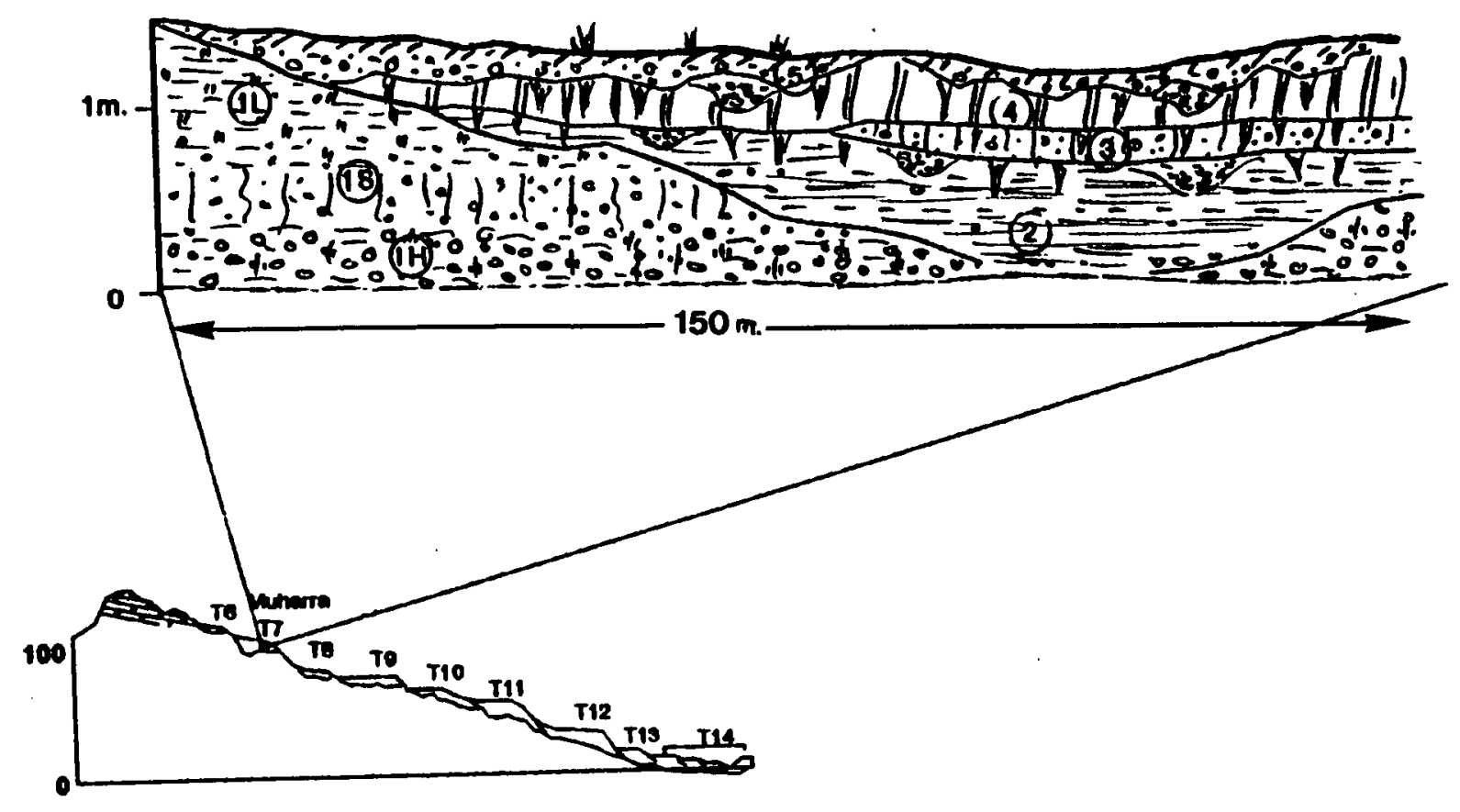

Coter Munura

Fig. 3: Posición geomorfológica de la Terraza de Muharra y secuencia estratigráfica. 1. Nivel de gravas basales: $1 \mathrm{H}$. Subnivel hidromorfo, 1S. Suelo rojo, 1L. Subnivel limo-arcilloso; 2. Depósito arcilloso de tonos verdes; 3 . Suelo pardorojizo; 4. Depósito arcilloso pardo-negruzco; 5. Suelo de cultivo. 

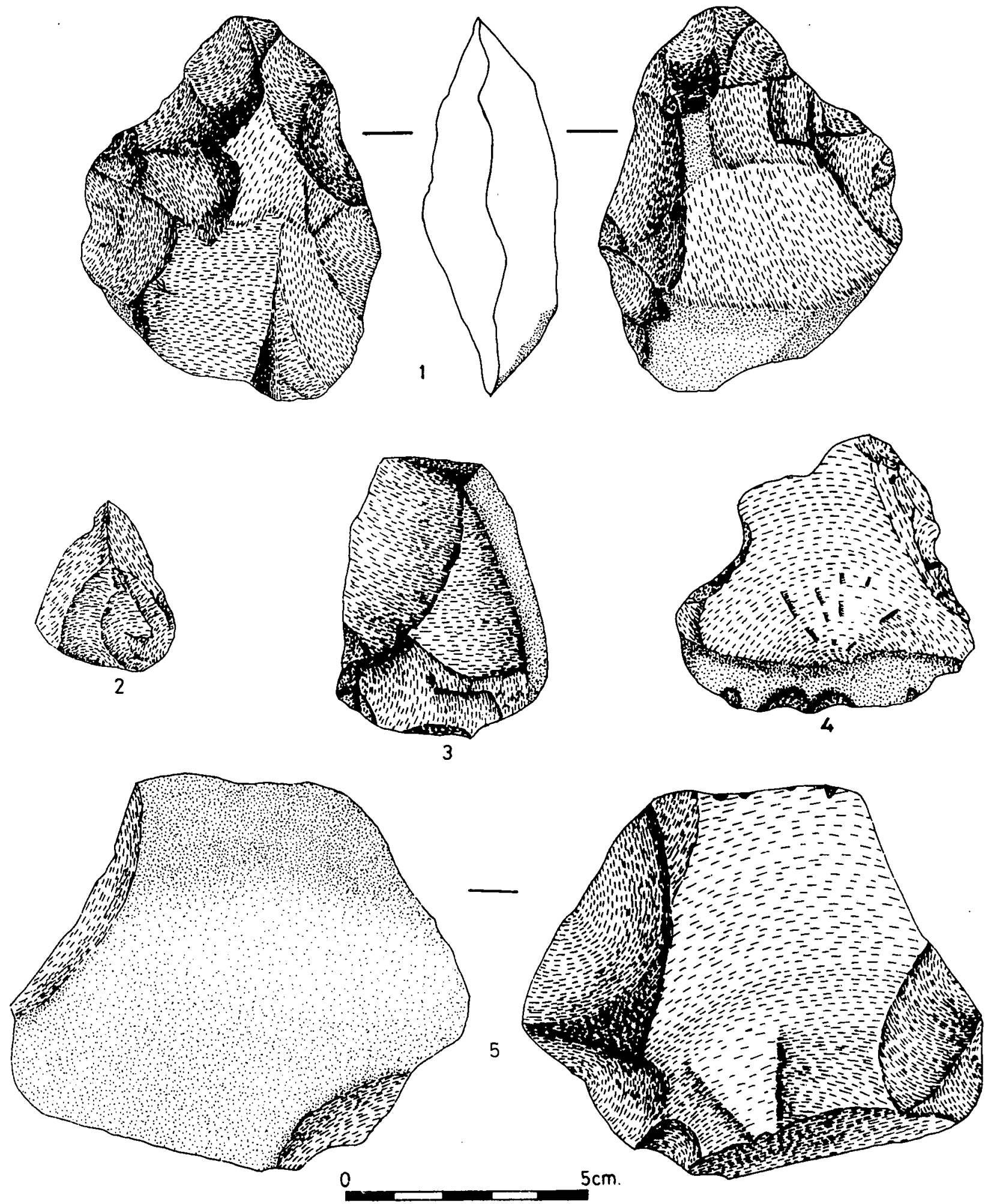

Fig. 4: 1. Bifaz del Nivel 3; 2. Punta levallois, 3. Cuchillo de dorso natural, 4. Muesca retocada, 5. Diverso (afín a Hendedor) del Nivel 1. 

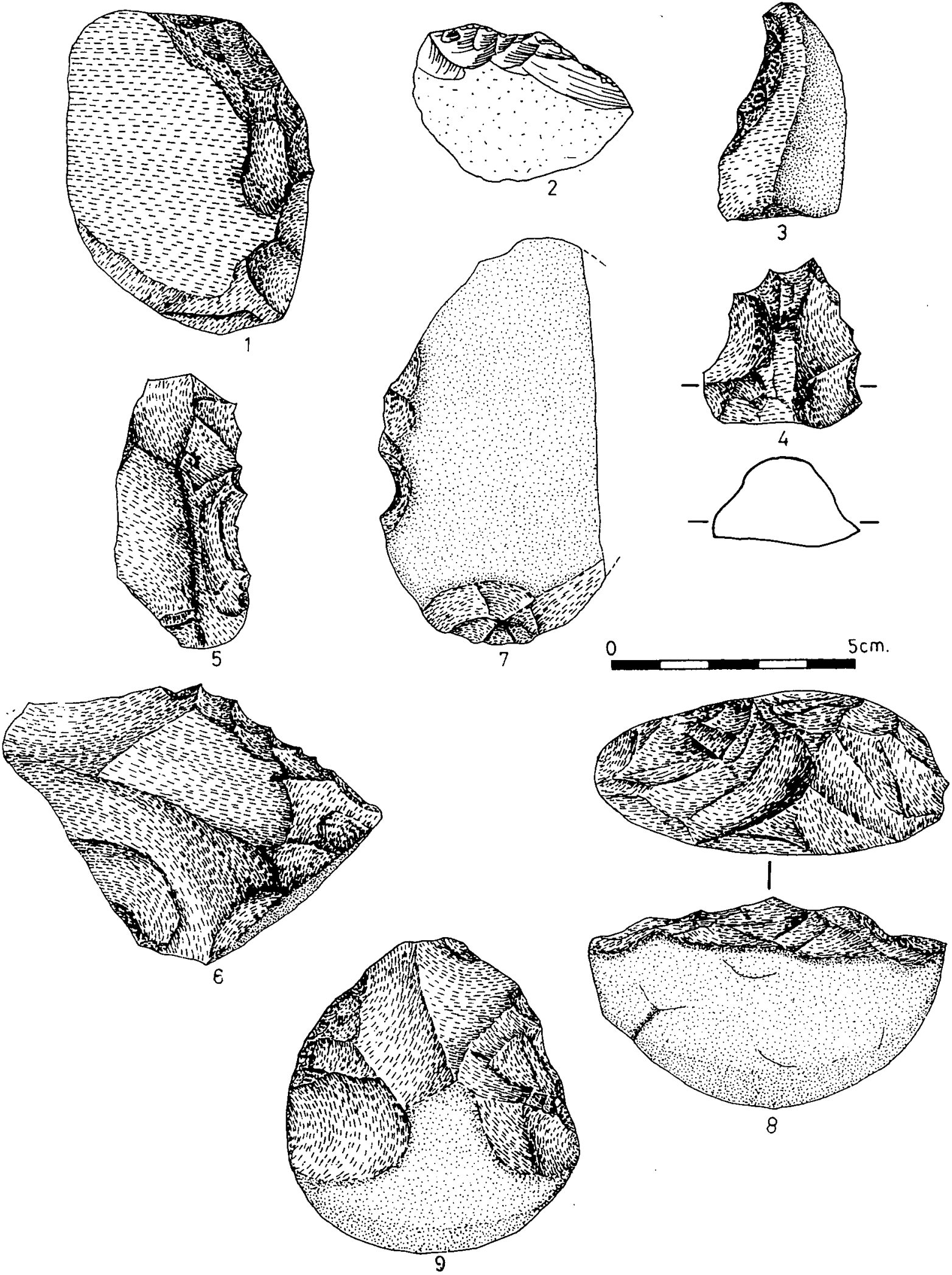

Fig. 5: Nivel 2: 1. Raedera sobre cara plana; 2. Raedera transversal; 3. Raedera cóncava; 4,5, y 6. Denticulados; 7. Muesca; 8. Canto truncado; 9. Canto tallado unifacial. 

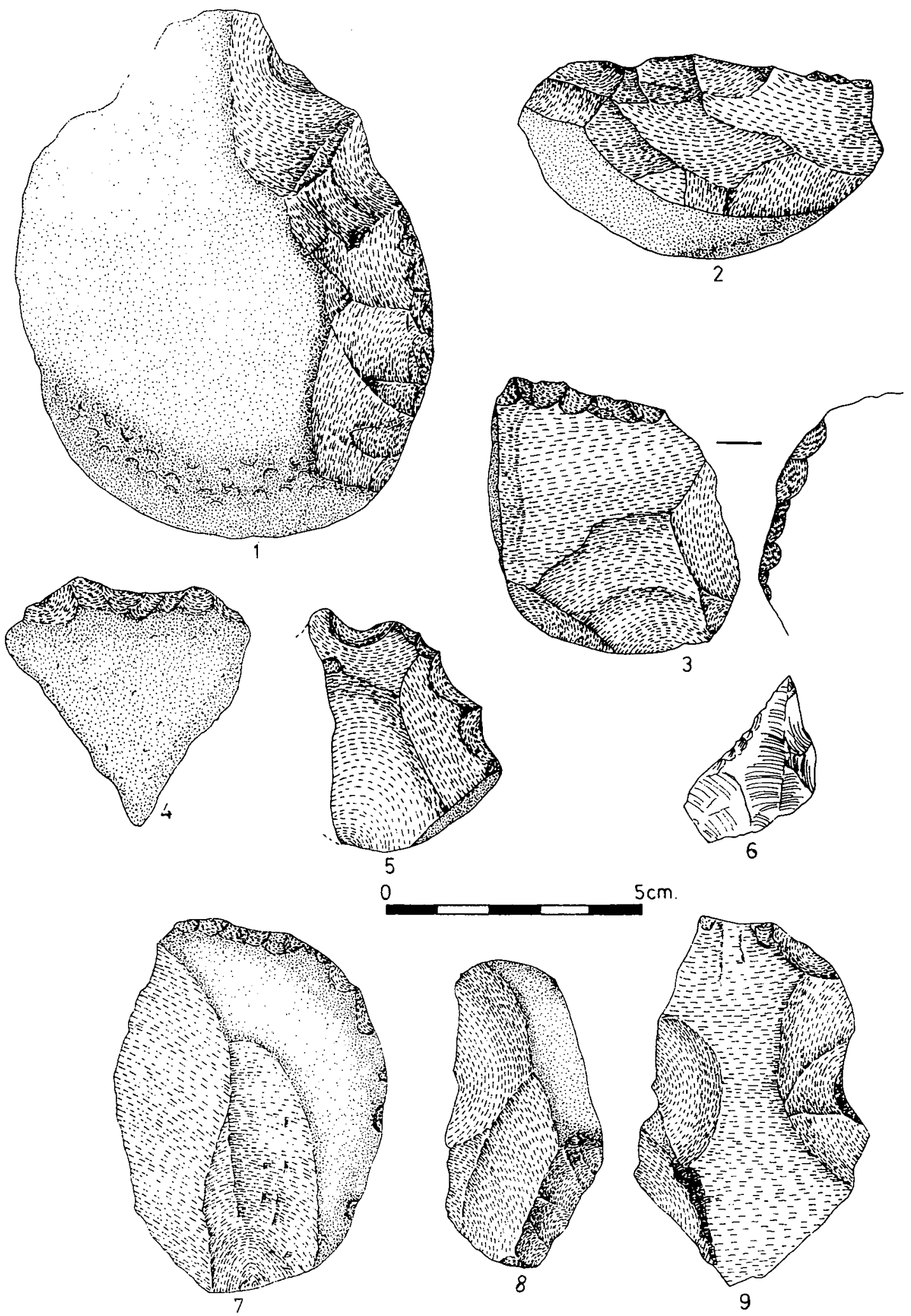

Fig. 6: Nivel 4: 1. Raedera convexa; 2. Raedera transversal; 3. Raedera alterna; 4. Raedera cóncava; 5. Denticulado; 6. Perforador atípico; 7 y 8 . Cuchillos de dorso atípico; 9. Muesca. 

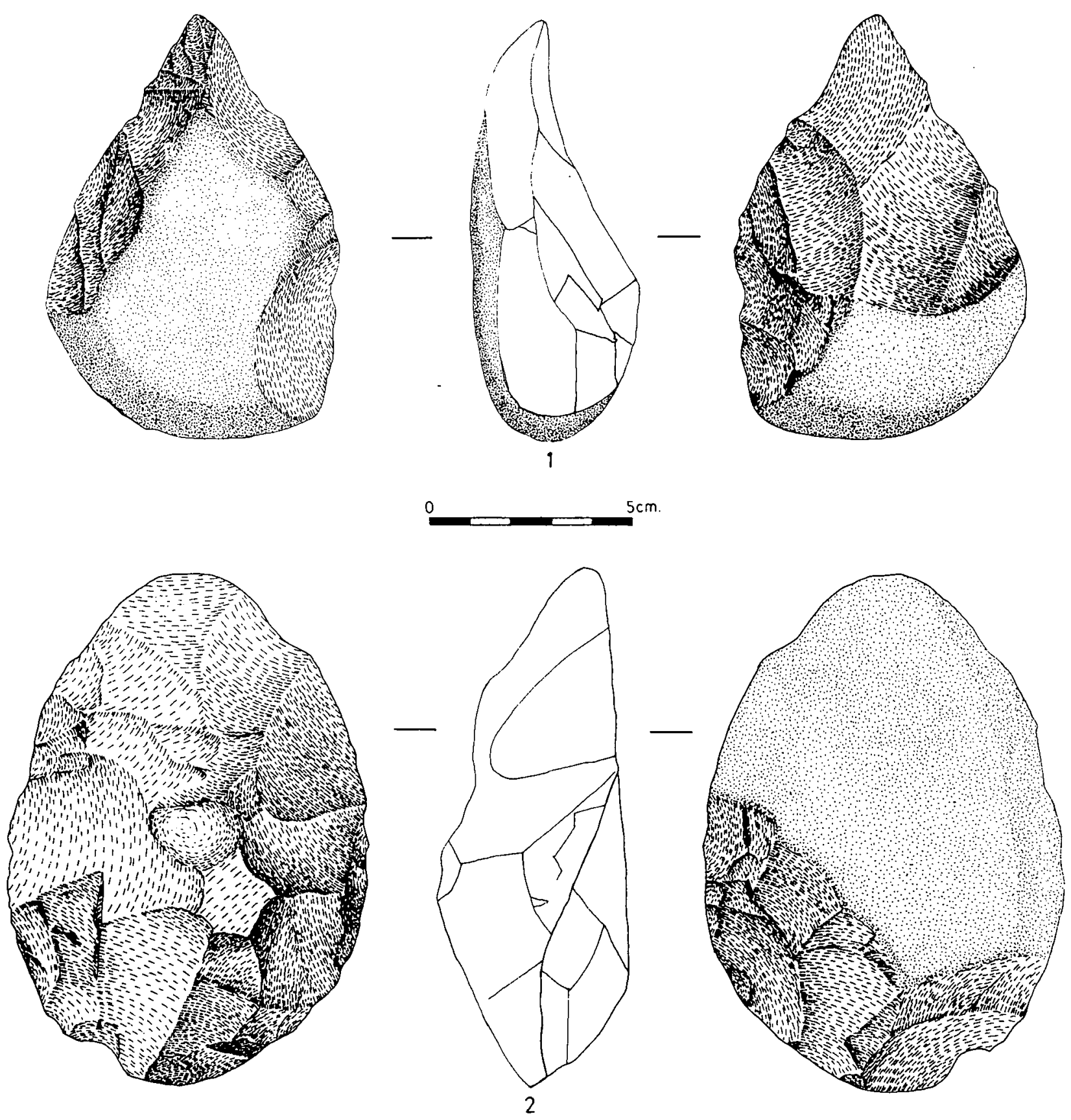

Fig. 7: Nivel 4: 1. Triedro; 2. Bifaz ovalado 


\begin{tabular}{|c|c|c|c|c|c|c|c|}
\hline \multirow[t]{3}{*}{ INDUSTRIA } & \multicolumn{3}{|c|}{ NIVEL 1} & & \multirow[t]{3}{*}{ NIVEL 2} & \multirow[t]{3}{*}{ NIVEL 3} & \multirow[t]{3}{*}{ NIVEL 4} \\
\hline & & $1 \mathrm{H}$ & & $1 S$ & & & \\
\hline & R3 & R2 & $\mathrm{R} 1$ & & & & \\
\hline \multicolumn{8}{|l|}{ NÚCLEOS } \\
\hline Extr. aisladas & 1 & 1 & & 1 & & 2 & 11 \\
\hline Extr. contiguas & & & 1 & 1 & 8 & & 13 \\
\hline Extr. bifaciles & & & & & & & 2 \\
\hline Poliédrico & & & & & 1 & & 1 \\
\hline Centrípedo & & & & & 1 & & 3 \\
\hline Lasca-núcleo & & & 1 & & & 1 & 1 \\
\hline Indefinido & & & & & 5 & 2 & 19 \\
\hline \multicolumn{8}{|l|}{ LASCA NO } \\
\hline \multicolumn{8}{|l|}{ RETOCADAS } \\
\hline Cortical & 3 & & 7 & & 6 & 2 & 21 \\
\hline Semicortical & & 1 & 8 & 2 & 11 & 1 & 39 \\
\hline Interna & & & 7 & & 4 & 5 & 50 \\
\hline De talla & & & 1 & & & & \\
\hline Debris y frag. & & & 19 & & 3 & 8 & 64 \\
\hline \multicolumn{8}{|l|}{ UTENSILIOS } \\
\hline L. Levallois & & & & & & 1 & 2 \\
\hline P. Levallois & & & 1 & & & & \\
\hline Raedera & & & & & 3 & & 12 \\
\hline Perforador & & & & & & & 3 \\
\hline C.D. atípico & & & & & & & 4 \\
\hline C.D. natural & & & 2 & & 1 & & 3 \\
\hline Muesca simple & & & 4 & & 6 & & 12 \\
\hline Muesca retocada & & & 1 & & 2 & & 2 \\
\hline Denticulado & & & & & 5 & & 3 \\
\hline Cepillo & & & & & 1 & & \\
\hline Canto Tallado & & & & & 2 & 1 & 5 \\
\hline Bifaz & & & & & & 1 & 1 \\
\hline Triedro & & & & & & & 1 \\
\hline \multirow[t]{3}{*}{ Diverso } & & & 1 & & & 1 & 1 \\
\hline & 4 & 2 & 53 & & & & \\
\hline & & 59 & & 4 & & & \\
\hline TOTAL & & 63 & & & 59 & 25 & 273 \\
\hline
\end{tabular}

Fig. 8: Resumen del conjunto lítico aparecido en la Terraza de Muharra. 